\title{
Methodik \\ der Wirtschaftsinformatik
}

\section{DOI 10.1007/s11576-010-0242-2}

\section{Die Autoren}

Prof. Dr. Robert Winter ( $\varangle$ )

Institut für Wirtschaftsinformatik

Universität St. Gallen

Müller-Friedberg-Strasse 8

9000 St. Gallen

Schweiz

Robert.Winter@unisg.ch

Prof. Dr. Richard Baskerville

Georgia State University

Atlanta

USA

Baskerville@acm.org

Online publiziert: 2010-08-25

This article is also available in English via http://www.springerlink.com and http://www.bise-journal.org: Winter R, Baskerville R (2010) Science of Business \& Information Systems Engineering. Bus Inf Syst Eng. doi: 10.1007/ s12599-010-0123-7.

(c) Gabler Verlag 2010 existierenden IS-Phänomenen sind. unterschiedlich hoch.
Im deutschsprachigen Raum dominiert die Gestaltungsorientierung als Forschungsansatz in der Wirtschaftsinformatik. Eine große Anzahl gestaltungsorientiert arbeitender Forschender findet sich aber auch in anderen, hauptsächlich europäischen Ländern. Ziele der gestaltungsorientierten Wirtschaftsinformatik sind die Entwicklung und Evaluation innovativer, nützlicher, übertragbarer Lösungen für wichtige und relevante ISGestaltungsprobleme in Wirtschaft und Verwaltung. Dadurch unterscheidet sie sich grundsätzlich von verhaltensorientierter Wirtschaftsinformatik, deren Ziele Ermittlung und Validierung kausaler, erklärender und/oder vorhersagender Beziehungen zwischen

Beide Forschungsansätze werden durch „Meta“-Forschung begleitet, welche die jeweiligen Prozesse, Ergebnisse, Rollen usw. erkenntnistheoretisch und/oder sozialwissenschaftlich reflektiert. Diese Reflektion dient zur Anleitung der Forschenden und definiert die Stringenz des jeweiligen Ansatzes. Gemeinsames Ziel aller Forschungsansätze ist die Verbindung von Stringenz und Relevanz. In der quantitativen, verhaltensorientierten Wirtschaftsinformatik haben sich bestimmte Metriken statistischer Signifikanz als klare und allgemein akzeptierte Stringenzmaße etabliert - allerdings ist die Relevanz der derart erarbeiteten Erkenntnisse unterschiedlich hoch. Im Gegensatz dazu hat sich in der gestaltungsorientierten Wirtschaftsinformatik die Nützlichkeit der erarbeiteten IS-basierten Lösungen wichtiger Probleme als allgemein akzeptiertes Relevanzmaß etabliert - allerdings ist die Stringenz der Konstruktion und Evaluation dieser Lösungen

Die Wirtschaftsinformatik adressiert die gesamte technoökonomische - und damit sowohl verhaltens- wie auch gestaltungsorientierte - Forschung, welche gleichzeitig methodisch fundiert sowie aus Sicht der betrieblichen Praxis relevant sein sollte. Aus Methodikperspektive der Wirtschaftsinformatik ist damit die Stringenz gestaltungsorientierter Wirtschaftsinformatik-Forschung ein wichtiges Thema. Diese ist offenbar weniger klar definiert und darüber herrscht weniger allgemeine Übereinkunft, als dies für die Stringenz verhaltensorientierter Forschung der Fall ist. Zwar gibt es viele Beiträge zur Rechtfertigung von Artefaktentwürfen, zur Typologie von Artefakten oder zu spezifischen Problemlösungen - jedoch sind Methodikfragen in der Forschendengemeinschaft nicht ausreichend standardisiert. Beispiele sind das Fehlen eines allgemein akzeptierten Referenz-Vorgehensmodells für gestaltungsorientierte WirtschaftsinformatikForschung oder die andauernde Debatte über die Positionierung und den Charakter von Design-Theorien. Obwohl Wirtschaftsinformatiker/innen sich darüber einig sind, dass Informationssysteme immer menschliche, aufgabenbezogene und (software-)technische Komponenten umfassen, ist umstritten, ob auch „reine“ Organisationslösungen ohne IT-Komponenten zulässige Forschungs- und Entwicklungsgegenstände sind. Ein weiterer Indikator für die Notwendigkeit einer stärkeren Hinwendung zu Stringenzfragen der gestaltungsorientierten Wirtschaftsinformatik ist das Fehlen allgemein akzeptierter Evaluationsrichtlinien für die verschiedenen Artefakttypen.

Dieses Schwerpunktheft widmet sich deshalb der Reflektion gestaltungsorientierter Wirtschaftsinformatik-Forschung und insbesondere Stringenzfragen der Artefaktkonstruktion. Der Aufruf zu Beiträgen wurde sowohl über die Kanäle der Zeitschrift wie auch über internationale Mailing-Listen verteilt. Interessierte Autoren/innen wurden dazu eingeladen, ihre Publikationsprojekte an einem eintägigen Workshop in St. Gallen im September 2009 vorzutragen und zu diskutieren. Diese Gelegenheit wurde von neun Autorenteams wahrgenommen; am Workshop nahmen außerdem zehn erfahrene Wirtschaftsinformatik-Forscher/innen teil. Zur Einreichungsfrist Anfang Dezember 2009 wurden 15 Arbeiten eingereicht, von denen für 13 aufgrund guter inhaltlicher Passung das Begutachtungsverfahren eröffnet wurde. Nach der ersten Begutachtungsrunde wurden fünf Beiträge abgelehnt; Acht Autorenteams wurde die Gelegenheit zur Überarbeitung und Wiedereinreichung gegeben. Nach der zweiten Begutachtungsrunde wurde eine Arbeit unter Bedingungen angenommen und vier Beiträge wurden zu einer weiteren Überarbeitung und Wiedereinreichung eingeladen. Nach der dritten und 
letzten Begutachtungsrunde wurde eine vorher unter Auflagen angenommene Arbeit final angenommen und wurden drei weitere Arbeiten unter Bedingungen angenommen. Insgesamt wurden damit vier Beiträge angenommen.

Es freut uns besonders, dass das in diesem Schwerpunktheft erscheinende WIInterview perfekt zu unserem Thema passt. Jay F. Nunamaker, einer der „Gründerväter" der Informationssystemforschung in den USA, beantwortet Robert Winters Fragen zur Zukunft der Informationssystemforschung, zu Stringenz- und Publikationsfragen gestaltungsorientierter Forschung sowie zur Organisation und Finanzierung von Informationssystemforschung.

Aufgrund von Längenbeschränkungen können leider nur drei der vier angenommenen Beiträge in diesem Schwerpunktheft publiziert werden:

Baskerville und Pries-Heje beteiligen sich an der Diskussion über Design-Theorien, in dem sie das fokussierte und vereinfachte Konzept einer erklärenden Design-Theorie vorschlagen. Erklärende Design-Theorien stellen Beziehungen zwischen Anforderungen und entsprechenden Problemlösungskomponenten her. Sie illustrieren ihren Vorschlag mit zwei Reihen von Beispielen aus verschiedenen Ingenieurdisziplinen sowie aus der Informationssystemforschung.

Offermann, Blom, Levina und Bub schlagen auf Grundlage des aktuellen Stands der Diskussion von Design-Theorien eine spezifische Design-Theorie für den Artefakttyp „Methode“ ab. Für jede Komponente des von ihnen favorisierten Design-TheorieVerständnisses entwickeln sie Evaluationskriterien und präsentieren dazu entsprechende Beispiele aus der Literatur.

Österle und Otto stellen eine Methode zur Konsortialforschung vor, durch welche Forschende und Unternehmen gestaltungsorientierte Forschung gemeinschaftlich durchführen können und damit sowohl Relevanz wie auch Stringenz von Forschungsergebnissen sicherstellen können. Diese Methode wurde über einen Zeitraum von zwanzig Jahren weiterentwickelt und evaluiert.

Der vierte Beitrag wird im Wirtschaftsinformatik-Heft 6/2010 erscheinen: Fettke, Houy und Loos betrachten die Rolle von Wissen im Prozess gestaltungsorientierter Wirtschaftsinformatik. Sie zeigen auf, dass Gestaltungswissen oft methodisch vernachlässigt wird, und präsentieren Grundlagen zur systematischen Erzeugung, Validierung und Weiter-/Wiederverwendung von Gestaltungswissen. Die Nützlichkeit ihres Vorschlags zeigen die Autoren exemplarisch am Beispiel ereignisgesteuerter Prozessketten.

Ein solches Schwerpunktheft wäre nicht ohne die Mitarbeit einer Vielzahl von Personen machbar. Wir möchten uns bei allen Autoren/innen, allen Workshopteilnehmern/innen und insbesondere allen Gutachtern/innen - sowohl aus dem deutschsprachigen Raum wie auch international - für ihre jeweiligen Beiträge bedanken, die zur erfolgreichen Realisierung dieses Schwerpunkthefts führten.

Robert Winter Richard Baskerville 